\title{
Host Genetic Effect on Deoxynivalenol Accumulation in Fusarium Head Blight of Barley
}

\author{
K. P. Smith, C. K. Evans, R. Dill-Macky, C. Gustus, W. Xie, and Y. Dong
}

First and fourth authors: Department of Agronomy and Plant Genetics, University of Minnesota, 411 Borlaug Hall, 1991 Upper Buford Circle, St. Paul 55108; and second, third, fifth, and sixth authors: Department of Plant Pathology, University of Minnesota, 495 Borlaug Hall, 1991 Upper Buford Circle, St. Paul 55108.

Accepted for publication 5 March 2004.

\section{ABSTRACT}

Smith, K. P., Evans, C. K., Dill-Macky, R., Gustus, C., Xie, W., and Dong, Y. 2004. Host genetic effect on deoxynivalenol accumulation in Fusarium head blight of barley. Phytopathology 94:766-771.

One of the major concerns with Fusarium head blight (FHB) of barley
is the potential health risks to livestock and humans through the
accumulation of the mycotoxin deoxynivalenol (DON) in infected grain.
To define the role of the host in DON accumulation during the early
stages of disease development, we conducted a series of greenhouse
experiments. We inoculated single spikelets of greenhouse-grown plants
with Fusarium graminearum, moved the plants to a dew chamber, and
harvested the inoculated spikelets after $72 \mathrm{~h}$ for DON analysis. We con-
ducted a quantitative trait locus (QTL) analysis using a genetic mapping population, constructed with the parents Stander and Frederickson, that segregated for DON accumulation after single-spikelet inoculation in two experiments. A single QTL on chromosome 3 explained 18 and $35 \%$ of the phenotypic variation in the two experiments. To validate this QTL for DON accumulation, we used a DNA marker to select near-isogenic lines from a family from the mapping population that was segregating at this QTL. Disease symptom development was similar between the nearisogenic lines; however, the mean DON concentration of the lines homozygous for the allele from the high DON parent was 2.5 -fold more than the lines homozygous for the alternate allele. A time course experiment showed that this effect on toxin accumulation was observed at 10 days post inoculation. The near-isogenic lines developed in this study should prove useful for further exploration of the role of DON in FHB.
Fusarium head blight (FHB), caused by Fusarium graminearum Schwabe (teleomorph: Gibberella zeae (Schwein.) Petch), often results in harvested grain contaminated with the mycotoxin deoxynivalenol (DON). The reemergence of this disease has devastated small grain production in the Upper Midwest (14) and caused tremendous economic losses to producers and rural communities in the region $(21,30)$. The presence of DON in either wheat or barley can impact how the grain will be used in the food system. Grain that is to be consumed by humans in one form or another must meet specific criteria with respect to DON concentration. Regulations for finished wheat products in the United States specify that DON concentrations be less than 1 ppm (9). The malting and brewing industries set stricter guidelines and will generally only purchase barley for malting that is less than $0.5 \mathrm{ppm}$ (14). In addition to perceived health risks, malt made from barley with high levels of DON also is correlated with a phenomenon known as gushing, which is undesirable in the brewing industry (24). Swine are particularly sensitive to DON (25), so regulations limit DON concentrations in grain to be fed to swine at $5 \mathrm{ppm}$, while the limit for ruminant animals is $10 \mathrm{ppm}$ (9).

There is some controversy over the role that DON plays in pathogenicity in the FHB interaction. Adams and Hart (1) evaluated the pathogenicity of $G$. zeae by protoplast fusion between toxigenic and nontoxigenic isolates and concluded that DON and 15-acetyldeoxynivalenol are neither pathogenicity factors nor virulence factors in Fusarium ear rot of maize. Because they did not include resistant check cultivars in their study, they could not investigate possible interactive effects of trichothecenes as pathogenicity and virulence factors (3). Arseniuk et al. (2) studied the

Corresponding author: K. P. Smith; E-mail address: smith376@umn.edu

Publication no. P-2004-0510-01R

(C) 2004 The American Phytopathological Society effects of trichothecene accumulation in relation to disease severity, kernel number per spike, kernel weight per spike, and 1,000kernel weight of wheat, triticale, and rye infected with an inoculum mixture of F. culmorum (Wm. G. Sm.) Sacc., F. avenaceum (Fr.:Fr.) Sacc. (teleomorph: G. avenacea R. J. Cook), and $F$ graminearum. Wheat suffered greater reductions in kernel weight and accumulated higher concentrations of DON. Arseniuk et al. (2) concluded that the system regulating DON accumulation in the host might be independent of FHB reaction because they observed that some susceptible host genotypes achieved high grain yield, although the kernels accumulated high levels of mycotoxins. Later investigations $(7,11,22)$ showed that $F$. graminearum strains with a disruption in the trichodiene synthase gene (Tri5), which disabled the synthesis of tricothecenes, were still pathogenic, but less virulent on wheat and other cereal host species compared with wild-type isolates.

The concentration of DON in grain generally has been correlated with disease severity. In several studies examining the inheritance of resistance to FHB in barley, correlation coefficients between FHB severity and DON concentration have ranged from 0.40 to $0.73(13,28,33)$. In mapping studies, many of the quantitative trait loci (QTLs) associated with FHB severity were coincident with QTLs for DON concentration and distributed across all seven chromosomes $(6,13,15,33)$. These results are expected, because the accumulation of DON in the kernel also would require successful infection and colonization of host tissue. Although it is clear that DON concentration levels are affected by FHB severity, it also is possible that host mechanisms for resistance to DON accumulation exist, acting independently of those for resistance to FHB. Indeed, a study using wheat near-isogenic lines found that some regions of the genome were associated with DON concentration but not FHB severity (32).

There is very little evidence for a host genetic effect directly on DON accumulation that is independent of FHB severity in small 
grains. Most studies that investigate resistance to DON accumulation use assays in which differences in DON concentration could result from differences in disease level. In the wheat study conducted by Zhou et al. (32), a single central spikelet was inoculated with $F$. graminearum and the entire spike assayed for DON concentration. Although this assay reduces variability in disease levels due to differences among host genotypes for resistance to initial infection, substantial differences in resistance to disease spread in the spike after initial infection could result in different levels of DON concentration. In wheat, resistance to spread is well known and the source of resistance used in the study by Zhou et al. (32), Sumai 3, carries a resistance allele at a major QTL for disease spread on chromosome 3BS (12). In contrast, little evidence for host variation for resistance to spread exists in barley, and most genotypes that have been investigated appear to have high resistance to spread compared with wheat $(5,27)$. A study using wheat suspension cultures to which labeled DON was added found that a resistant cultivar was able to degrade DON faster than a susceptible cultivar (18). Measuring DON concentration in inoculated spikelets is one means of directly assessing the host effect on accumulation of DON independently of resistance to initial infection. An examination of barley lines using this approach found that there appeared to be differences among genotypes for DON accumulation in spikelets (8). Interestingly, many of the cultivars that had resistance to FHB in the field accumulated higher levels of DON after single-spikelet inoculation.

Increased understanding of the role of the host in DON accumulation associated with FHB will be essential to elucidate the host-pathogen interaction and develop strategies to minimize the impact of FHB on the yield and quality of barley. Phenotypic differences in DON accumulation first were observed between cvs. Frederickson and Stander by Evans et al. (8). The objectives of this study were to determine if there is a genetic basis in the host for DON accumulation by mapping QTLs for DON concentration in the Frederickson $\times$ Stander population and to characterize the dynamics of toxin accumulation in selected barley genotypes.

\section{MATERIALS AND METHODS}

Mapping population and near-isogenic lines. The mapping population was derived from a cross between Frederickson (CIho 13647) and Stander (PI 564743) and was the basis of previous research to study genetic resistance to FHB (15). Frederickson is a two-rowed barley cultivar with moderate resistance to FHB and Stander is a FHB-susceptible six-rowed cultivar (23). The population consisted of $130 \mathrm{~F}_{4}$-derived recombinant inbred lines (RILs). The linkage map included 143 simple sequence repeat (SSR) and restriction fragment length polymorphism (RFLP) markers distributed throughout the genome.

Near-isogenic lines were developed for a region on chromosome 3 from a single line in the mapping population that was sixrowed and segregating for the region containing the SSR marker Bmac0067. Seventy seed from this family were planted for each of two greenhouse experiments. Each plant was genotyped with Bmac0067 and individual plants that were either homozygous for the Frederickson allele (FF) or homozygous for the Stander allele (SS) were used in the greenhouse experiment and single-spikelet inoculated with $F$. graminearum as described below. Twenty-four plants from each marker class (SS and FF) were used for each experiment.

DON assay of spikelets from inoculated greenhouse-grown plants. Plants were grown in pots containing commercial potting medium (Metromix 200; Scott-Sierra Horticultural Products Co., Marysville, $\mathrm{OH}$ ) in a greenhouse maintained at 20 to $25^{\circ} \mathrm{C}$. Each pot was fertilized with $3 \mathrm{~g}$ of slow-release 14-14-14 (N-P-K) fertilizer. Plants were inoculated when at least one spike from each plant had fully emerged from the boot. A single isolate of F. graminearum (Butte86ADA-11) was used to produce macro- conidia inoculum (8). Plants were inoculated using a repeating 500- $\mu$ l Hamilton 700 series syringe (model 80830) with PB600 dispenser (model 83700). Inoculum (10 $\mu$ l containing $\approx 1,000$ macroconidia) was injected into a central spikelet of a single barley spike of each plant. Immediately after inoculation, plants were moved to a dew chamber maintained at $21^{\circ} \mathrm{C}$ with a relative humidity near $100 \%$ and continuous light provided by two $40-\mathrm{W}$ fluorescent tubes $\left(6 \mu \mathrm{mol} \mathrm{m} \mathrm{m}^{-2} \mathrm{~s}^{-1}\right)$. For the QTL mapping and validation experiments, inoculated spikelets were harvested at 72 $\mathrm{h}$ postinoculation (PI).

For each harvested spikelet sample, we estimated the percentage of surface tissue of the palea and lemma that exhibited chlorosis and necrosis. Inoculated spikelets were removed from the spike using forceps that were washed in acetone before harvesting each sample and placed in vials and frozen at $-80^{\circ} \mathrm{C}$ until needed for toxin analyses. Toxin analyses of single-spikelet extracts for the detection and quantification of DON were made following the procedures of Mirocha et al. (20) with the following modifications. Single spikelets were extracted by soaking and shaking with acetonitrile/water $(84 / 16 \mathrm{vol} / \mathrm{vol})$ for $24 \mathrm{~h}$. A temperature program was used for gas chromatography-mass spectrometry analysis with an initial column temperature of $150^{\circ} \mathrm{C}$ for $1 \mathrm{~min}$, and then increased to $280^{\circ} \mathrm{C}$ at a rate of $30^{\circ} \mathrm{C} / \mathrm{min}$ and held for $5 \mathrm{~min}$. The injection temperature was kept at $300^{\circ} \mathrm{C}$, and column flow rate was $1 \mathrm{ml} / \mathrm{min}$. Selected ion monitoring was applied to detect the characteristic ions of DON with fragment ion $(\mathrm{m} / \mathrm{z}$ value) of 235.10 as target ion and 259.10 and 422.10 as reference ions. Following extraction of DON from spikelets, vials were left uncapped and placed in a drying oven at 40 to $50^{\circ} \mathrm{C}$. After drying for $24 \mathrm{~h}$, spikelets were weighed to determine dry weight of each sample. The dry spikelet weight was used to quantify concentration of DON in each of the inoculated spikelets.

QTL analysis. The data from the mapping population was analyzed as a completely randomized design with five and six replicates for experiments 1 and 2, respectively. Analysis of variance was conducted on data for the RIL population as individual experiments and pooled across experiments using Proc GLM (SAS Institute, Cary, NC). The parents, Frederisckson and Stander, were compared using a $t$ test. To identify QTL associated with DON accumulation, we conducted composite interval mapping (CIM) analyses using the software package PLABQTL (29) with the following options: additive genetic model, cov select, Fto-enter $=3.5$, RAIC $=3$, and scanning interval of 1 centimorgan. PLABQTL uses a stepwise regression procedure to select cofactors and between six and eight cofactors were selected for each of our analyses. We report QTL detected with a LOD score $>3.42$, corresponding to $P<0.05$ and $P<0.0004$ experiment-wise and comparison-wise error rate, respectively, as calculated by the $\chi^{2}$ approximation suggested by Zeng (31). We estimated the proportion of phenotypic variance explained by each QTL using the coefficient of determination $\left(R^{2}\right)$, which is based on the partial correlation of a putative QTL with the trait adjusted for cofactors in the multilocus model. For each QTL, the average effect of an allele $(\alpha)$ from Fredrickson was calculated as the regression coefficient for the corresponding QTL genotype in a multilocus regression model, assuming no dominance.

Validation of DON accumulation QTL on chromosome 3. The near-isogenic lines (described above) were planted in two greenhouse experiments. Lines were grown in the greenhouse in a completely randomized design and inoculated as previously described. Plants were sampled for DON analysis $72 \mathrm{~h}$ PI. At sampling, the percentage of tissue exhibiting chlorosis and necrosis was visually estimated. We used unpaired $t$ tests to compare means of near-isogenic lines with contrasting alleles at the chromosome 3 QTL.

Toxin time-course experiment. Six six-rowed RILs from the mapping population were selected for further study in a toxin time-course experiment. Three of these RILs accumulated DON 
at low concentrations (FEG8-35, FEG8-66, and FEG8-87) and three RILs accumulated DON at high concentrations (FEG8-01, FEG8-19, and FEG8-58) based on the mapping experiments. In addition, the genotype of the low lines was SS at the DON accumulation QTL on chromosome 3, while the genotype of the high lines was FF. The time-course experiments were planted and grown as described previously and arranged as a completely randomized design with six genotypes, seven time points, and three replicates. Inoculated spikelets were sampled immediately following inoculation, and at 18, 36, 54, and $72 \mathrm{~h}$ during the dew period; then, plants were returned to greenhouse benches after $72 \mathrm{~h}$ and sampled at 120 and $240 \mathrm{~h}$ PI. Concentration of DON and disease symptoms were quantified as previously described. Analysis of variance was conducted, using Proc GLM, on the DON concentration data with experiment, time point, genotype (FF or SS), and RIL as factors. In addition, individual analyses were conducted to determine whether the genotype effect was significant at each time point.

\section{RESULTS}

Mapping QTL for accumulation of DON. Two greenhouse experiments were conducted to assess DON concentration in the parents, Frederickson and Stander, and the RILs of the mapping population $72 \mathrm{~h}$ PI. As in previous experiments, the moderately FHB-resistant parent, Frederickson, accumulated higher levels of DON compared with the FHB-susceptible parent, Stander, although these two parents were significantly different only in experiment 1 (Table 1). In the combined analysis, the line-byexperiment interaction was not significant $(P=0.56)$ for DON concentration. Analysis of individual experiments revealed significant variation among RILs for DON concentration $(P<$ 0.001 ). Frederickson had $\approx 20 \%$ higher spikelet dry weight than Stander. This was expected because Frederickson is two-rowed and Stander is six-rowed, and two-rowed cultivars generally produce larger spikelets. The line-by-experiment interaction for the population was significant for spikelet weight; therefore, results from only the individual experiments are presented. Spikelet weight was significantly different among the RILs in both experiments $(P<0.001)$.

Composite interval mapping analysis identified two QTLs associated with DON accumulation in RILs of the FredericksonStander mapping population. The largest QTL effect is located on chromosome 3 and was detected in both experiments (Table 2). This QTL was located near the centromere of chromosome 3 between the markers Bmac0122 and Bmac0067 (15), which is in the region of BIN 6 on the Steptoe $\times$ Morex barley BIN map. The Frederickson allele at this locus was associated with higher DON concentration. A second putative QTL, located on chromosome 4, was detected only in experiment 1 . At this locus, the Frederickson allele also was associated with higher DON. Because DON concentration was calculated using spikelet weight, we also conducted a QTL analysis of dried spikelet weight. A single major QTL for spikelet dry weight was detected at the Vrs1 locus on chromosome 2, which controls two-rowed or six-rowed spike morphology (data not shown). The two QTL regions associated with DON accumulation were not associated with spikelet weight.

Validation of DON accumulation QTL on chromosome 3. To independently validate the QTL on chromosome 3, we selected a six-rowed $\mathrm{F}_{4: 7}$ line from the Frederickson-Stander mapping population that was segregating for the SSR marker Bmac0067 at the QTL. We examined all of the marker genotype data for this line, FEG8-14, and determined that it was fixed for 132 of the 143 marker loci included in the map and segregating for 11 marker loci located in four regions of the genome, including the QTL region on chromosome 3. Marker loci were fixed for the other DON accumulation QTL region on chromosome 4 . We considered plants from this line that were genotyped as either FF (Fredrickson allele) or SS (Stander allele) at the QTL with marker Bmac0067 to be near-isogenic, because they were selected from a set of $\mathrm{F}_{4: 7}$ lines from which the $\mathrm{F}_{4}$ plant was fixed at most other locations in the genome. The mean DON concentration of the lines with the FF genotype was $\approx 2.5$-times higher than those with

TABLE 1. Parent means and recombinant inbred line population means and ranges for deoxynivalenol (DON) concentration and spikelet weight $\mathrm{y}^{\mathrm{y}}$

\begin{tabular}{|c|c|c|c|c|c|}
\hline \multirow[b]{2}{*}{ Population $^{\mathrm{Z}}$} & \multicolumn{3}{|c|}{ DON (ppm) } & \multicolumn{2}{|c|}{ Spikelet weight (mg) } \\
\hline & Experiment 1 & Experiment 2 & Two-experiment mean & Experiment 1 & Experiment 2 \\
\hline \multicolumn{6}{|l|}{ Parents } \\
\hline Frederickson & $60.7 \mathrm{a}$ & $30.8 \mathrm{a}$ & $45.8 \mathrm{a}$ & $6.2 \mathrm{a}$ & $4.3 \mathrm{a}$ \\
\hline \multicolumn{6}{|l|}{ Population } \\
\hline Minimum & 1.8 & 1.6 & 7.9 & 2.4 & 1.6 \\
\hline Maximum & 166.3 & 148.8 & 158.3 & 9.7 & 8.0 \\
\hline
\end{tabular}

y Parent values followed by the same letter are not significantly $(P<0.05)$ different as determined by $t$ test.

${ }^{\mathrm{z}} \mathrm{LSD}=$ least significant difference.

TABLE 2. Chromosome (Chr.) location, percent phenotypic variation $\left(R^{2}\right)$, and average effect of a gene substitution $(\alpha)$ for deoxynivalenol (DON) accumulation quantitative trait loci (QTLs) identified by composite interval mapping (CIM) analysis in two greenhouse experiments with the Fredrickson $\times$ Stander mapping population

\begin{tabular}{|c|c|c|c|c|c|c|c|c|c|c|c|c|c|}
\hline \multirow[b]{2}{*}{ Marker interval $^{u}$} & \multirow[b]{2}{*}{ Chr. } & \multicolumn{4}{|c|}{ Experiment 1} & \multicolumn{4}{|c|}{ Experiment 2} & \multicolumn{4}{|c|}{ Two-experiment mean } \\
\hline & & $\mathrm{cM}^{\mathrm{v}}$ & $\mathrm{LOD}^{\mathrm{w}}$ & $R^{2 \mathrm{x}}$ & $\alpha(\mathrm{ppm})^{\mathrm{y}}$ & $\mathrm{cM}$ & LOD & $R^{2}$ & $\alpha(\mathrm{ppm})$ & $\mathrm{cM}$ & LOD & $R^{2}$ & $\alpha(\mathrm{ppm})$ \\
\hline Bmag0122-Bmac0067 & 3 & 0 & 16.1 & 35.1 & 19.6 & 0 & 4.4 & 17.6 & 10.2 & 0 & 8.0 & 25.8 & 11.4 \\
\hline Bmag0306-HVM068 & 4 & 26 & 4.6 & 2.5 & 4.4 & $\ldots$ & $\ldots$ & $\ldots$ & $\ldots$ & $\ldots$ & $\ldots$ & $\ldots$ & $\ldots$ \\
\hline Full model ${ }^{z}$ & $\ldots$ & $\ldots$ & 12.1 & 36.0 & $\ldots$ & $\ldots$ & 4.4 & 17.6 & $\ldots$ & $\ldots$ & $\ldots$ & $\ldots$ & $\ldots$ \\
\hline
\end{tabular}

u Markers flanking the QTL LOD scan peak. Marker positions are those published by Mesfin et al. (15).

$\checkmark$ Position of QTL peak in centimorgans.

${ }^{w}$ A LOD score of 3.4 was the threshold for detection of a QTL.

x Partial $R^{2} \times 100$.

${ }^{y}$ Effect of the Frederickson allele calculated as the regression coefficient for the corresponding QTL genotype in the multi-locus regression model.

${ }^{\mathrm{z}}$ Multiple regression model. 
the SS genotype (Table 3). This confirms the results of the mapping experiment because the $\alpha$ values (Table 2) can be used to predict the two genotypic classes by adding and subtracting $\alpha$ from the population mean (Table 1). These estimates predict that individuals with the genotypes FF and SS would have 61.5 and $22.3 \mathrm{ppm}$ of DON in experiment 1 (2.8-fold) and 42.5 and $22.1 \mathrm{ppm}$ of DON in experiment 2 (1.9-fold), respectively. There was no difference in percent necrosis at $72 \mathrm{~h}$ PI on inoculated spikelets between the two marker genotype classes (Table 3). Percent chlorosis differed only in experiment 2, where the SS class had a higher percentage of chlorosis on inoculated spikelets while still having significantly lower toxin levels.

Toxin time-course experiment. Three barley genotypes from the mapping population that accumulated high levels of DON and three that accumulated low levels of DON were selected to investigate changes in toxin accumulation between 0 and $240 \mathrm{~h}$ PI (Table 4). Water-soaking on the palea and lemma of inoculated spikelets was first observed at $36 \mathrm{~h}$ PI. Both water-soaking and chlorosis were observed at $54 \mathrm{~h} \mathrm{PI}$, with chlorosis covering 10 to $75 \%$ of palea and lemma tissues on nearly all of the inoculated spikelets. Necrosis on spikelets first was observed at $72 \mathrm{~h}$ PI and covered from 10 to $100 \%$ of the palea and lemma tissue. However, a majority of spikelets also were exhibiting extensive chlorosis that ranged from 10 to $100 \%$. There were practically no escapes from infection by $72 \mathrm{~h}$ PI. At $120 \mathrm{~h}$ PI, most of the spikelets were showing complete necrosis with only a few spikelets exhibiting only chlorotic palea and lemma tissue.

The analysis of variance indicated significant RIL-by-experiment interaction; therefore, the data for DON accumulation are presented as separate experiments (Fig. 1). DON accumulation first was detected at $36 \mathrm{~h} \mathrm{PI}$ and generally increased to the end of

TABLE 3. Disease symptoms (chlorosis and necrosis) and deoxynivalenol (DON) content in spikelets $72 \mathrm{~h}$ postinoculation for near-isogenic lines for a DON accumulation quantitative trait locus on chromosome 3 linked to simple-sequence repeat marker Bmac0067

\begin{tabular}{lrcr}
\hline & \multicolumn{3}{c}{ Bmac0067 genotype $^{\mathrm{y}}$} \\
\cline { 2 - 4 } Experiment, phenotype & FF & SS & $P^{\mathrm{z}}$ \\
\hline 1 Chlorosis (\%) & 41.9 & 43.1 & \\
Necrosis (\%) & 15.4 & 19.4 & 0.89 \\
DON (ppm) & 101.0 & 43.3 & $<0.58$ \\
2 Chlorosis (\%) & & & \\
Necrosis (\%) & 30.2 & 53.7 & $<0.01$ \\
DON (ppm) & 52.6 & 42.4 & 0.33 \\
\hline
\end{tabular}

${ }^{\mathrm{y}}$ In all, 24 barley lines were selected as being homozygous Frederickson (FF) or homozygous Stander (SS) for the simple-sequence repeat marker Bmac0067 in each of two experiments.

${ }^{\mathrm{z}}$ Probability from unpaired $t$ test.

TABLE 4. Deoxynivalenol (DON) concentration after single-spikelet inoculation for three low and three high DON-accumulating lines selected from the Frederickson $\times$ Stander mapping population based on two greenhouse experiments

\begin{tabular}{lcc}
\hline & \multicolumn{2}{c}{ DON concentration $(\mathrm{ppm})^{\mathrm{z}}$} \\
\cline { 2 - 3 } RIL $^{\mathrm{y}}$ & Experiment 1 & Experiment 2 \\
\hline FEG8-01 & 166 & 149 \\
FEG8-19 & 93 & 97 \\
FEG8-58 & 154 & 63 \\
FEG8-35 & 9 & 18 \\
FEG8-66 & 14 & 6 \\
FEG8-87 & 13 & 10 \\
LSD (0.05) & 68 & 49
\end{tabular}

${ }^{\mathrm{y}}$ Recombinant inbred line from the Frederickson $\times$ Stander mapping population. LSD = least significant difference.

${ }^{\mathrm{z}}$ Parts per million DON detected in inoculated single-spikelet $72 \mathrm{~h}$ postinoculation in the quantitative trait locus mapping experiment. the experiment at $240 \mathrm{~h}$ PI (Fig. 1). Throughout the experiments, the genotypes selected as accumulating high levels of DON generally had higher DON levels than the lines selected originally for low DON accumulation The differences between high and low lines were significantly different at $72 \mathrm{~h}$ PI in experiment 1 and 72 and $120 \mathrm{~h} \mathrm{PI}$ in experiment 2 . The line FEG8-01 consistently was the highest in terms of DON accumulation, whereas FEG887 generally was the lowest (Table 4; Fig. 1). The line FEG8-58, selected as a genotype that accumulated high levels of DON, varied considerably between the two experiments (Fig. 1).

\section{DISCUSSION}

This study has documented and genetically mapped a host effect on DON accumulation in barley following inoculation with $F$. graminearum. The assay that we used permitted us to separate the host effect on initial infection and spread of disease from the accumulation of DON. In the Frederickson $\times$ Stander genetic study by Mesfin et al. (15), the region of chromosome 3 containing the DON accumulation QTL reported here was not associated with either FHB severity or DON concentration in field experiments; however, it was associated with FHB severity in one of two greenhouse experiments. Interestingly, the allele of the FHBsusceptible parent (Stander) was associated with a lower percentage of spikelets infected, indicating that this allele actually conferred some reduction in disease severity. In this study, the Stander allele at the DON accumulation QTL on chromosome 3 was associated with lower DON concentration; however, all the inoculated spikelets showed the classic symptoms of infection by $F$. graminearum, indicating that successful infection had occurred. In addition, near-isogenic lines for the DON accumulation QTL

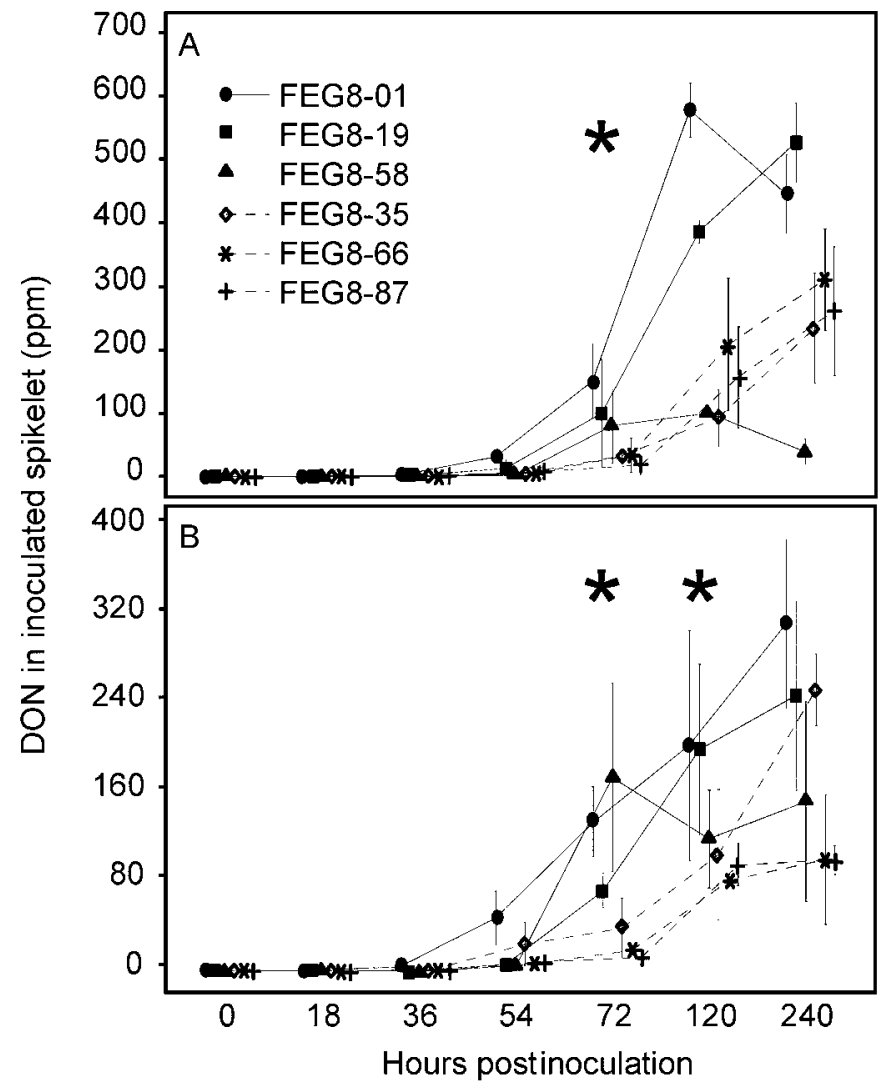

Fig. 1. Accumulation of deoxynivalenol (DON) from 0 to $240 \mathrm{~h}$ postinoculation for three high and three low DON-accumulating barley lines from the Frederickson $\times$ Stander mapping population. $\mathbf{A}$ and $\mathbf{B}$ are experiments 1 and 2, respectively. Error bars are the standard error of the mean. An asterisk above the time point indicates that the difference between the high and low lines was significant $(P<0.05)$ at that time. 
were not significantly different in the extent of chlorosis that developed on inoculated spikelets at $72 \mathrm{~h}$ PI. In one of the two single-spikelet inoculation experiments, the extent of necrosis was higher in the lines carrying the Stander allele. Because the Stander allele at this locus is not associated with reduced disease symptoms resulting from single-spikelet inoculation, but is associated with lower percent spikelet infection following sprayinoculation, this allele may act to lower the probability of successful infection by reducing the accumulation of DON. Desjardins et al. (7) observed that wheat plants inoculated with isolates of $F$. graminearum that did not produce tricothecenes had lower disease incidence compared with wild-type trichothecene-producing strains, suggesting that DON plays a role in establishing infection.

Although it is clear that the Stander allele at the QTL on chromosome 3 conditions lower DON accumulation under controlled conditions, the mechanism or mechanisms by which this occurs is not. At least three possible mechanisms exist. One is that alleles at this locus are involved in a mechanism to degrade DON. Miller et al. (19) proposed the degradation of DON as a mechanism of resistance to FHB in wheat and later showed that wheat suspension cultures could degrade DON (18). Another possibility is that the Stander allele has the effect of restricting growth of the pathogen after initial infection, thus lowering toxin production. Several studies with wheat have shown a significant positive correlation between fungal biomass, as estimated by ergosterol (ERG) concentration, and DON concentration $(16,17)$. A third possibility is that fungal growth is equal between lines carrying either allele at the QTL, but that production of DON by the fungus is lower in lines carrying the Stander allele. In studies conducted with wheat, there is no consistent association between the quantitative expression of DON and disease. The ratio of ERG to DON concentrations in infected spikelets were reported as similar (26) and different $(16,19)$ among resistant and susceptible wheat lines. Furthermore, no association was found between ERG/DON ratios and aggressiveness among 42 strains of $F$. culmorum inoculated on rye (10).

A key question is whether the host effect on DON accumulation changes when the host-pathogen interaction transitions from parasitic to saprophytic. Our study focused on host genetic effects of this interaction in the early stages of disease development. We have not yet tested these near-isogenic lines in a field experiment to determine whether the host effect on DON accumulation several days after infection translates into lower levels of DON in ripe grain at harvest. In the study conducted by Mesfin et al. (15), there was no evidence for a QTL for DON concentration in harvested grain at the chromosome 3 QTL reported here (LOD values were less than 1.0). Although this study indicates that host genetic mechanisms to modify DON concentration are independent of resistance to disease severity, in the field studies using the Frederickson $\times$ Stander mapping population there was a significant positive correlation between FHB severity and DON concentration in harvested grain, and resistant lines accumulated less toxin (15). Similarly, in wheat inoculated with F. culmorum, there was a strong correlation between DON concentration and FHB severity in spikelets at 8 weeks after inoculation, but not at 4 weeks after inoculation (26). The transition from parasitic to saprophytic interaction could alter host genetic effects on toxin accumulation during pathogenesis and parasitism, particularly if the mechanisms leading to lower DON concentrations require living host cells.

One concern with QTL mapping experiments is lack of power to detect QTLs and the possibility of bias in estimating the magnitude of effects of alleles at QTLs (4). In both experiments in this study, the QTL detected explained less than $40 \%$ of the phenotypic variation. Furthermore, the quantitative nature of inheritance of resistance to DON accumulation and wide segregation for this trait in the mapping population suggests the involvement of more than two genes. Therefore, it is likely that other genes that affect DON accumulation were not detected due to experimental error in estimating DON concentrations, poor marker coverage in some regions of the genome, or lack of statistical power due to the size of the mapping population. Nevertheless, the estimates of the allelic effect of the chromosome 3 QTL based on the mapping experiment are similar to estimates derived from the validation experiment.

The characterization of a host genetic effect on DON accumulation and development of near-isogenic lines should prove useful to define more precisely the role of DON and possibly other trichothecenes in this host-pathogen interaction. It would be interesting to determine, for example, if host genetic differences in DON concentration are the result of differences in growth of the pathogen, production of toxin, or host-mediated degradation of toxin. The utility of the DON accumulation QTL to develop new cultivars with lower levels of DON is not clear. Cv. Frederickson, which is a source of resistance to $\mathrm{FHB}$, carries the allele for higher DON accumulation. Further research is necessary to determine if there are other alleles at this locus that condition even lower rates of DON accumulation than the Stander allele, and whether the effect that we observed on accumulation of DON in single spikelets translates to lower DON levels in harvested grain. If host genetic factors affect FHB severity and DON accumulation independently, then resistance screening efforts should take into account disease level and DON concentration separately, to fully exploit the potential of both resistance mechanisms. Elucidating the host role in DON accumulation resulting from FHB offers an alternative avenue of research that should complement ongoing research efforts to manage this disease.

\section{ACKNOWLEDGMENTS}

This work was supported, in part, through grants provided by the U.S. Wheat and Barley Scab Initiative and the Minnesota Small Grains Initiative. We thank A. Elakkad, K. Wennberg, E. Schiefelbein, G. Velasquez, and P. Warnke for technical assistance in conducting greenhouse experiments; B. Scanlon for processing grain samples for DON analyses; and J. Anderson and B. Steffenson for providing critical reviews of an earlier draft of this manuscript.

\section{LITERATURE CITED}

1. Adams, G. C., and Hart, L. P. 1989. The role of deoxynivalenol and 15acetyldeoxynivalenol in pathogenesis by Gibberella zeae, as elucidated through protoplast fusions between toxigenic and nontoxigenic strains. Phytopathology 79:404-408.

2. Arseniuk, E., Foremska, E., Goral, T., and Chelkowski, J. 1999. Fusarium head blight and accumulation of deoxynivalenol (DON) and some of its derivatives in kernels of wheat, triticale and rye. J. Phytopathol. 147:577590 .

3. Atanasov, Z., Nakamura, C., Mori, N., Kaneda, C., Kato, H., Jin, Y. Z., Yoshizawa, T., and Murai, K. 1994. Mycotoxin production and pathogenicity of Fusarium species and wheat resistance to Fusarium head blight. Can. J. Bot. 72:161-167

4. Beavis, E. E. 1998. QTL analysis: power, precision and accuracy. Pages 145-162 in: Molecular Dissection of Complex Traits. A. H. Paterson, ed. CRC Press, Boca Raton, FL.

5. Cappetini, F. 1999. Inheritance of resistance to Fusarium Head Blight in barley. Ph.D. thesis. University of Minnesota, UMI 9941711.

6. de la Peña, R. C., Smith, K. P., Capettini, F., Muehlbauer, G. J., Gallo Meagher, M., Dill Macky, R., Somers, D. A., and Rasmusson, D. C. 1999. Quantitative trait loci associated with resistance to Fusarium head blight and kernel discoloration in barley. Theor. Appl. Genet. 99:561-569.

7. Desjardins, A. E., Proctor, R. H., Bai, G., McCormick, S. P., Shaner, G., Buechley, G., and Hohn, T. M. 1996. Reduced virulence of trichothecenenonproducing mutants of Gibberella zeae in wheat field tests. Mol. PlantMicrobe Interact. 9:775-781.

8. Evans, C. K., Xie, W., Dill-Macky, R., and Mirocha, C. J. 2000. Biosynthesis of deoxynivalenol in spikelets of barley inoculated with macroconidia of Fusarium graminearum. Plant Dis. 84:654-660.

9. FAO. 1997. Food and Nutrition Paper 64, Worldwide regulations for mycotoxins 1995. FAO, Rome.

10. Gang, G., Miedaner, T., Schumacher, U., and Geiger, H. H. 1998. Deoxy- 
nivalenol production by Fusarium culmorum isolates differing in aggressiveness toward winter rye. Phytopathology 88:879-884.

11. Harris, L. J., Desjardins, A. E., Plattner, R. D., Nicholson, P., Butler, G., Young, J. C., Weston, G., Proctor, R. H., and Hohn, T. M. 1999. Possible role of trichothecene mycotoxins in virulence of Fusarium graminearum on maize. Plant Dis. 83:954-960.

12. Kolb, F. L., Bai, G. H., Muehlbauer, G. J., Anderson, J. A., Smith, K. P., and Fedak G. 2001. Host plant resistance genes for Fusarium head blight: Mapping and manipulation with molecular markers. Crop Sci. 41:611-619.

13. Ma, Z., Steffenson B. J., Prom L. K., and Lapitan N. L. V. 2000. Mapping quantitative trait loci for Fusarium head blight resistance in barley. Phytopathology 90:1079-1088.

14. McMullen, M. P., Jones, R., and Gallenberg, D. 1997. Scab of wheat and barley: A re-emerging disease of devastating impact. Plant Dis. 81:13401348.

15. Mesfin, A., Smith, K. P., Dill-Macky, R., Evans, C. K., Waugh, R., Gustus, C. D., and Muehlbauer, G. J. 2003. Quantitative trait loci for Fusarium head blight resistance in barley detected in a two-rowed by sixrowed population. Crop Sci. 43:307-318.

16. Miedaner, T., Reinbrecht, C., Lauber, U., Schollenberger, M., and Geiger, H. H. 2001. Effects of genotype and genotype-environment interaction on deoxynivalenol accumulation and resistance to Fusarium head blight in rye, triticale, and wheat. Plant Breed. 120:97-105.

17. Miedaner, T., Reinbrecht, C., and Schilling, A. G. 2000. Association among aggressiveness, fungal colonization, and mycotoxin production of 26 isolates of Fusarium graminearum in winter rye head blight. J. Plant Dis. Prot. 107:135-144.

18. Miller, J. D., and Arnison, P. G. 1986. Degradation of deoxynivalenol by suspension cultures of the Fusarium head blight resistant cultivar Frontana. Can. J. Plant Pathol. 8:147-150.

19. Miller, J. D., Young, J. C., and Sampson, D. R. 1985. Deoxynivalenol and Fusarium head blight resistance in spring cereals. Phytopathol. Z. 113:359-367

20. Mirocha, C. J., Kolaczkowski, E., Xie, W., Yu, H., and Jelen, H. 1998. Analysis of deoxynivalenol and its derivatives (batch and single kernel) using gas chromatography/mass spectrometry. J. Agric. Food Chem. 46:1414-1418.

21. Nganje, W. E., Johnson, D. D., Wilson, W. W., Leistritz, F. F., Bangsund, D. A., and Tiapo, N. M. 2001. Economic impacts of Fusarium head blight in wheat and barley: 1998-2000. Agribusiness and Applied Economics Report No. 464 October 2001.
22. Proctor, R. H., Hohn, T. M., and McCormick, S. P., 1995. Reduced virulence of Gibberella zeae caused by disruption of a trichothecene toxin biosynthetic gene. Mol. Plant-Microbe Interact. 8:593-601.

23. Rasmusson, D. C., Wilcoxson, R. D., and Wiersma, J. V. 1993. Registration of 'Stander' barley. Crop Sci. 33:1403.

24. Schwarz, P. B., Beattie, S., and Casper, H. H. 1996. Relationship between Fusarium infestation of barley and the gushing potential of malt. J. Inst. Brew. 102:93-96.

25. Smith, T. K., McMillan, E. G., and Castillo, J. B. 1997. Effect of feeding blends of Fusarium mycotoxin-contaminated grains containing deoxynivalenol and fusaric acid on growth and feed consumption of immature swine. J. Anim. Sci. 75:2184-2191.

26. Snidjers, C. H. A., and Krechting, C. F. 1992. Inhibition of deoxynivalenol translocation and fungal colonization in Fusarium head blight resistant wheat. Can. J. Bot. 70:1570-1576.

27. Steffenson, B. J. 2002. Fusarium head blight of barley: Impact, epidemics, management, and strategies for identifying and utilizing genetic resistance. Pages 241-295 in: Fusarium Head Blight of Wheat and Barley. K. L. Leonard and W. R. Bushnell, eds. The American Phytopathological Society, St. Paul, MN

28. Urrea, C. A., Horsley, R. D., Steffenson, B. J., and Schwarz, P. B. 2002. Heritability of Fusarium head blight resistance and deoxynivalenol accumulation from barley accession Ciho 4196. Crop Sci. 42:14041408.

29. Utz, H. F., and Melchinger, A. E. 1996. PLABQTL: A program for composite interval mapping of QTL. Published online by J. Quant. Trait Loci.

30. Windels, C. E. 2000. Economic and social impacts of Fusarium head blight: Changing farms and rural communities in the northern great plains. Phytopathology 90:17-21.

31. Zeng, Z. B. 1994. Precision mapping of quantitative trait loci. Genetics 136:1457-1468

32. Zhou, W. C., Kolb, F. L., Bai, G. H., Domier, L. L., and Yao, J. B. 2002. Effect of individual Sumai 3 chromosomes on resistance to scab spread within spikes and deoxynivalenol accumulation within kernels in wheat. Hereditas 137:81-89.

33. Zhu, H., Gilchrist, L., Hayes, P., Kleinhofs, A., Kudrna, D., Liu, Z., Prom, L., Steffenson, B., Toojinda, T., and Vivar, H. 1999. Does function follow form? Principal QTLs for Fusarium head blight are coincident with QTLs for inflorescence traits and plant height in a doubled-haploid population of barley. Theor. Appl. Genet. 99:1221-1232. 